\title{
Selective Removal of Metallic Single-Walled Carbon Nanotubes with Small- Diameters by Using Nitric and Sulfuric Acids
}

\author{
Cheol-Min Yang ${ }^{\dagger}$, Jin Sung Park ${ }^{\dagger}$, Kay Hyeok An ${ }^{\dagger}$, Seong Chu Lim ${ }^{\dagger}$, Kwanyong Seo ${ }^{\ddagger}$, Bongsoo Kim ${ }^{\ddagger}$, Kyung Ah Park ${ }^{\dagger}$, Seungwu Han ${ }^{\S}$, Chong Yun \\ Park $^{\dagger}$, and Young Hee Lee ${ }^{*}$,
}

BK21 Physics Division, Institute of Basic Science, Center for Nanotubes and Nanostructured Composites, Sungkyunkwan University, Suwon 440-746, Republic of Korea,

Department of Chemistry, Korea Advanced Institute of Science \& Technology, 373-1, Guseong-dong, Yuseong-gu, Daejeon 305-701, Republic of Korea,

Department of Physics, Division of Nano Science, Ewha Womans University, Seoul 120-750, Republic of Korea 


\section{Supporting Information-Figure S1}
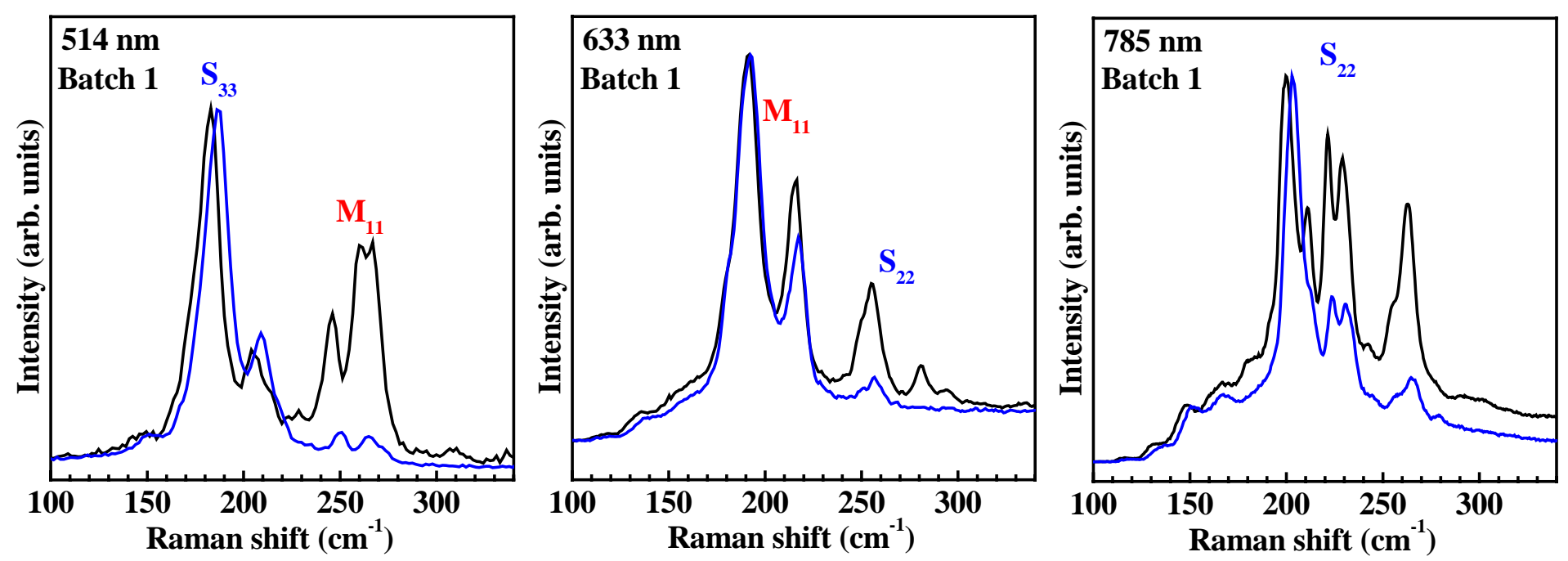

Figure S1. RBM Raman spectra for batch 1 HiPco SWCNTs at 514, 633, and $785 \mathrm{~nm}$ excitation wavelengths. The black and blue lines are from the pristine and air-oxidized HiPco SWCNTs (at $350{ }^{\circ} \mathrm{C}$ in air atmosphere). $\mathrm{M}_{\mathrm{ii}}$ and $\mathrm{S}_{\mathrm{ii}}$ correspond to metallic and semiconducting interband transitions. Gas-phase oxidation treatment led to diameter-selective removal of SWCNTs, regardless of metallicity.

\section{Fig. S1. Yang et al.}




\section{Supporting Information-Figure S2}

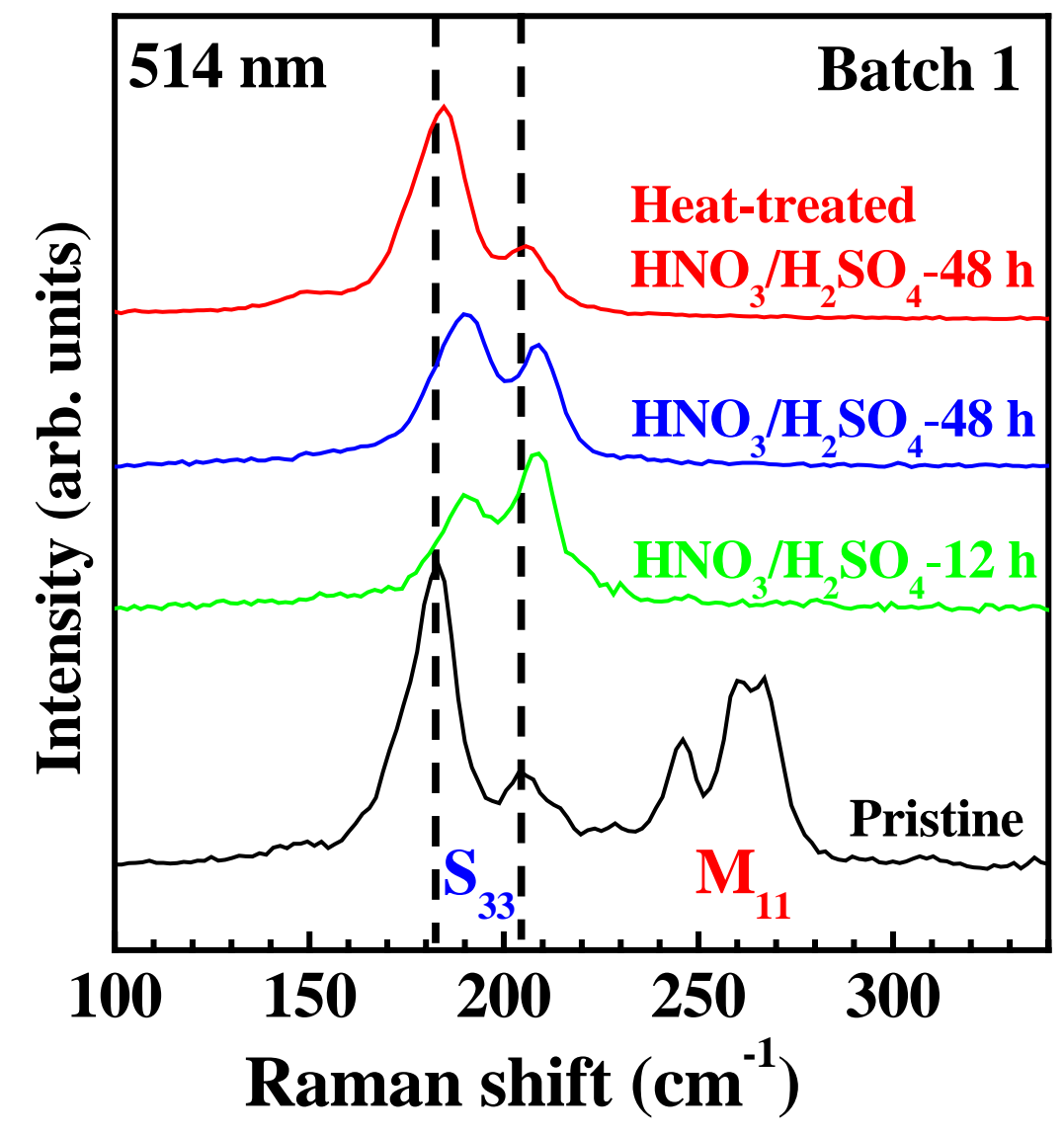

Figure S2. RBM Raman spectra from the leftover on the filter for batch 1 HiPco SWCNTs at $514 \mathrm{~nm}$ excitation wavelength. The black, green, blue, and red lines are from the pristine, $\mathrm{HNO}_{3} / \mathrm{H}_{2} \mathrm{SO}_{4}(1: 9)$ treatment for $12 \mathrm{~h}, 48 \mathrm{~h}$, and $\mathrm{HNO}_{3} / \mathrm{H}_{2} \mathrm{SO}_{4}$ (1:9) treatment for $48 \mathrm{~h}$ followed by heat treatment at $900{ }^{\circ} \mathrm{C}$ in $\mathrm{Ar}$ atmosphere, respectively. The peak shift was recovered after the heat treatment.

Fig. S2. Yang et al. 


\section{Supporting Information-Figure S3}

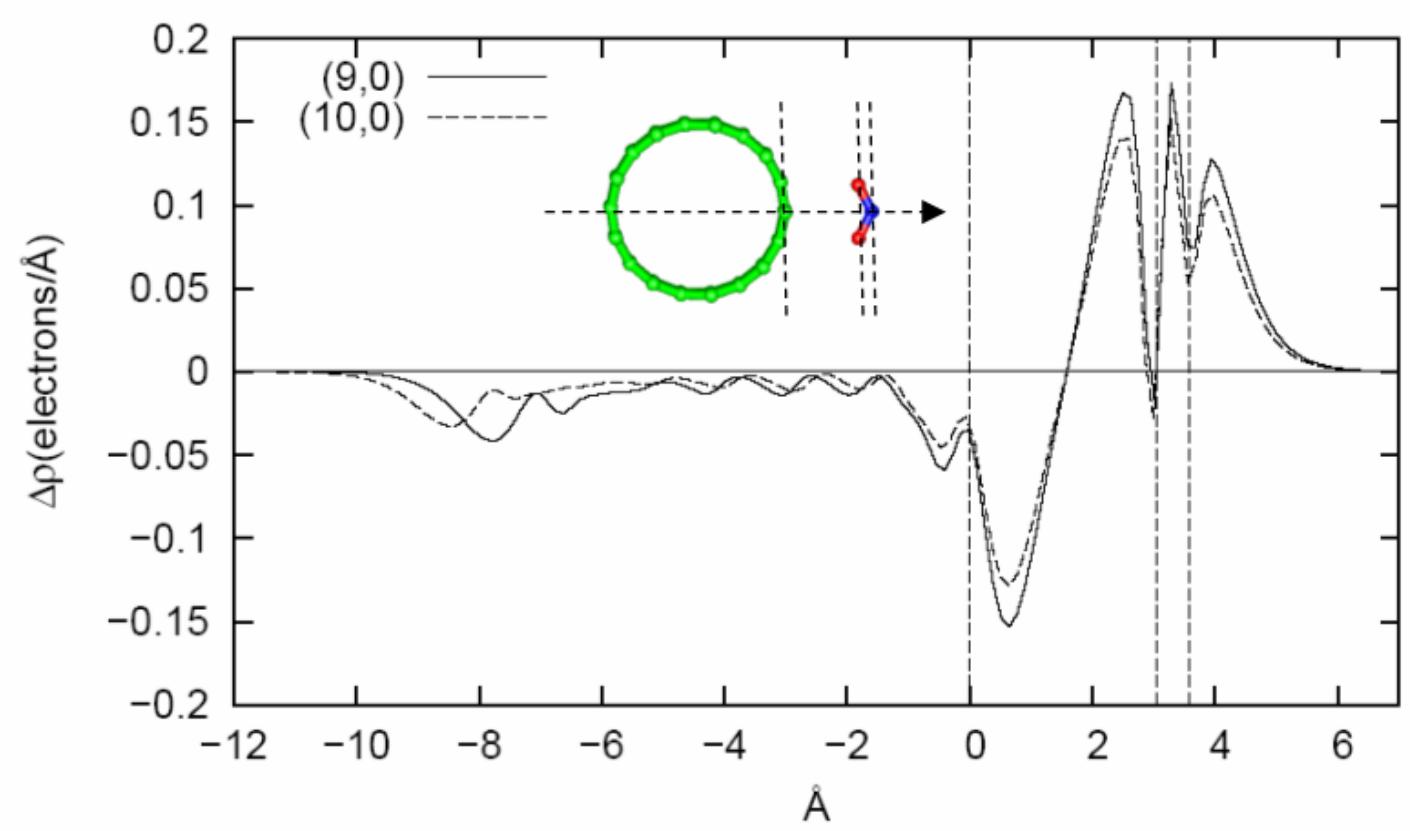

Figure S3. The redistribution of electronic density was calculated by $\Delta \rho=\rho\left(\mathrm{CNT}+\mathrm{NO}_{2}\right)-\rho(\mathrm{CNT})-\rho\left(\mathrm{NO}_{2}\right)$.

The figure was drawn along the arrowed direction shown in the inset figure and $\Delta \rho$ is averaged over the plane perpendicular to the plotting direction. Three vertical lines in the inset and figure indicate the positions of the outermost $\mathrm{C}, \mathrm{O}$, and $\mathrm{N}$ atoms, respectively. The enhanced charge transfer of metallic $(9,0)$ nanotube was clearly visible.

\section{Fig. S3. Yang et al.}

